Agr. Biol. Chem., 39 (5), 953 958, 1975

\title{
Hydroxylation of 2H-1,4-Benzodiazepine-2-ones by Streptomyces Species
}

\author{
Fumitaka Kishimoto, Kazuko Suzuki, Shigeo Ogino and Hisao Yamamoto \\ Research Department, Pharmaceutical Division of Sumitomo Chemical Co., Ltd., \\ Takarazuka, Hyogo Prefecture, Japan \\ Received August 26, 1974
}

\begin{abstract}
About 400 identified and unidentified Streptomyces stock cultures were examined for their capacity to hydroxylate benzodiazepines. Ten cultures (Streptomyces sp. S-3, S-4, S-12, S-19, S-60, S-120, S-145, St. flavus ATCC 3369, St. griseochromogeneus ATCC 14,511 and St. chartreusis ATCC 14,922) were found to hydroxylate $2 \mathrm{H}-1,4$-benzodiazepine-2-ones in the 3-position of the seven-membered ring. The transformation products were isolated and identified.

The culture conditions of Streptomyces sp. S-145 for the production of 7-chloro-1,3dihydro-3-hydroxy-5-(2'-fluorophenyl)-2H-1,4-benzodiazepine-2-one (IIIb) were studied.
\end{abstract}

Microbial transformation of benzodiazepines has not been extensively studied. A patent $^{13}$ concerning the process for microbial modification of benzodiazepines by fermentation with Pellicularia filamentosa was issued in 1969. It has shown that three strains of $P$. filamentosa (f. sp. microsclerotia CBS, f. sp. microsclerotia IFO 6298 and f. sp. sasakii IFO 6675) hydroxylate 7-chloro-1, 3-dihydro-1methyl-5-phenyl-2H-1, 4-benzodiazepine-2-one (diazepam, Ia) and desmethyl diazepam (IVa) in the 3-position of the seven-membered ring. The transformation of benzodiazepines by microorganisms other than $P$. filamentosa, however, has not been reported.

It is the purpose of this investigation to ascertain whether a similar reaction occurs in microorganisms other than $P$. filamentosa.

\section{MATERIALS AND METHODS}

Microorganisms. About 400 identified and unidentified Streptomyces were used.

Chemicals. Benzodiazepines used for substrate and authentic substances were synthesized according to the methods cited in the literature. ${ }^{2 \sim 4}$ ?

The chemical structures of $2 \mathrm{H}-1$,4-benzodiazepine-2ones are as follows.

Media. The following four media were used for this investigation. SM-1 medium (for screening and transformation): glucose, $5 \%$; yeast extract, $0.5 \%$; soy bean meal, $0.5 \% ; \mathrm{KH}_{2} \mathrm{PO}_{4}, 0.1 \% ; \mathrm{K}_{2} \mathrm{HPO}_{4}, 0.2 \%$ and $\mathrm{FeSO}_{4} \cdot 7 \mathrm{H}_{2} \mathrm{O}, 0.1 \%$ (pH 7.0). SM-2 medium (for transformation): glucose, $1 \%$; peptone, $0.2 \%$; meat

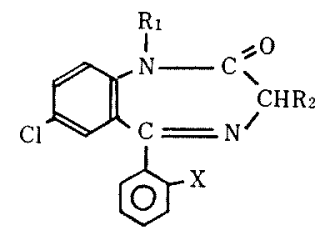

$$
\begin{aligned}
& \text { I a : } \mathrm{R}_{1}=\mathrm{CH}_{3}, \quad \mathrm{R}_{2}=\mathrm{X}=\mathrm{H} \text { (diazepam) } \\
& \text { b : } \mathrm{R}_{1}=\mathrm{CH}_{2}-\mathrm{R}, \mathrm{R}_{2}=\mathrm{X}=\mathrm{H} \quad \text { (prazepam) } \\
& \text { c: } \mathrm{R}_{1}=\mathrm{CH}_{3}, \quad \mathrm{R}_{2}=\mathrm{H}, \mathrm{X}=\mathbf{F} \\
& \text { II a : } \mathrm{R}_{1}=\mathrm{CH}_{3}, \quad \mathrm{R}_{2}=\mathrm{OH}, \quad \mathrm{X}=\mathrm{H} \\
& \mathrm{b}: \mathrm{R}_{1}=\mathrm{CH}_{2}-\mathrm{J}, \mathrm{R}_{2}=\mathrm{OH}, \mathrm{X}=\mathrm{H} \\
& \text { c: } \mathrm{R}_{1}=\mathrm{CH}_{3}, \mathrm{R}_{2}=\mathrm{OH}, \mathrm{X}=\mathrm{F} \\
& \text { III }: \mathrm{R}_{1}=\mathrm{X}=\mathrm{H}, \quad \mathrm{R}_{2}=\mathrm{OH} \quad \text { (oxazepam) } \\
& \mathrm{b}: \mathrm{R}_{1}=\mathrm{H}, \quad \mathrm{R}_{2}=\mathrm{OH}, \quad \mathrm{X}=\mathrm{F} \\
& c: \mathrm{R}_{1}=\mathrm{H}, \mathrm{R}_{2}=\mathrm{OH}, \mathrm{X}=\mathrm{Cl} \text { (lorazepam) } \\
& \mathrm{Na}: \mathrm{R}_{1}=\mathrm{R}_{2}=\mathrm{X}=\mathrm{H} \\
& \mathrm{b}: \mathrm{R}_{1}=\mathrm{R}_{2}=\mathrm{H}, \quad \mathrm{X}=\mathrm{F} \\
& \therefore \mathrm{R}_{1}=\mathrm{R}_{2}=\mathrm{H}, \quad \mathrm{X}=\mathrm{Cl} \\
& \text { (Chemical structure) }
\end{aligned}
$$

extract, $0.1 \%$; yeast extract, $0.1 \%$ and glycine, $0.05 \%$ (pH 7.0). SM-3 medium (for transformation): glucose, $1 \%$; soy bean meal, $1.5 \%$ and $\mathrm{NaCl}, 0.5 \%$ (pH 7.0). SM-4 medium (for preservation of cultures): glucose, $1 \%$; peptone, $0.2 \%$; meat extract, $0.1 \%$; yeast extract, $0.1 \%$; benzodiazepine, $0.1 \%$ and agar.

Screening method. A loopful of cells was transferred from agar slants into a test tube containing $15 \mathrm{ml}$ of the SM-1 medium. The tubes were incubated at $28^{\circ} \mathrm{C}$ on a reciprocal shaker until adequate growth was obtained. To each tube, $0.15 \mathrm{ml}$ of alcohol solution of benzodiazepine $(100 \mathrm{mg} / \mathrm{ml})$ was added and incubation was further continued. After 2, 4 and 6 days incubation, aliquot portions were removed aseptically and extracted 
with the same volume of chloroform.

The chloroform extracts and reference compounds (1-methyl-oxazepam, IIa; desmethyl diazepam, IVa and oxazepam, IIIa) were spotted on glass plates coated with silica gel containing fluorescent indicator (Merk, Silica Gel HF, $250 \mu$ in thickness). The plates were developed in a solvent system of chloroform-acetoneethanol $(17: 2: 1, v / v)$. The chromatograms were visualized under long and shortwave ultraviolet light before and after spraying with $2 \mathrm{~N} \mathrm{H}_{2} \mathrm{SO}_{4}$. Phenolic products were detected with ferric chloride-ferricyanide reagent.

Cultures capable of forming products identical with or similar to IIa and IIIa or forming ferric chlorideferricyanide positive products were stocked on agar slant (SM-4 medium).

Cultivation. Seed culture, previously grown for 2 to 4 days, was inoculated either into a $500 \mathrm{ml}$ Sakaguchi flask containing $100 \mathrm{ml}$ of medium or into a 10 liter jar fermentor containing 6 liters of medium. The inoculum size was two per cent $(\mathrm{v} / \mathrm{v})$. Sakaguchi flasks were incubated at $28^{\circ} \mathrm{C}$ on a reciprocal shaker. Seven liters of air per min wereintroduced into the jar fermentor, After incubation at $28^{\circ} \mathrm{C}$ for 1 to 2 days, a $5 \%$ solution of benzodiazepine in ethanol was added to a final concentration of $0.1 \%$ and further incubation was carried out for 6 to 7 days.

Isolation of products. The culture broth was filtrated and extracted three times with a half volume of chloroform. The chloroform layer was evaporated to dryness under vacuum.

The compounds shown with the structure I were isolated by silica gel column chromatography (Kanto Chemical Co., Ltd., 100 200 mesh) with the solvent system of chloroform-acetone-ethanol (17:2:1).

The compounds shown with the structure II were isolated by silica gel column chromatography, followed by preparative thin-layer chromatography (Merk, Silica Gel HF, $500 \mu$ in thickness) with the same solvent system described above.

The compounds shown with the structure III and IV were isolated by silica gel column chromatography and/ or Sephadex LH-20 gel filtration (Pharmacia Co.).

Sephadex LH-20 gel filtration. Chloroform solution of the products was applied on Sephadex LH-20 column previously swollen with chloroform and elution was carried out with chloroform. The compound I, II and IV were eluted in this order. After complete elution of the compound IV, the compound III was eluted by feeding ethanol.

Identification. For structural studies of products, ultraviolet, infrared and nuclear magnetic resonance spectroscopies were used. UV, IR and NMR spectra were obtained with Shimadzu MPS-50L, Hitachi EPIG3 and Varian T-60, respectively.
Melting points were determined with Yanagimoto micro melting-point apparatus and all melting points were uncorrected.

Analytical procedures. Quantitative estimation of Ic, IVb and IIIb in the fermentation broth was performed by gas chromatography.

Two milliliters of sample taken out from the fermentation broth was extracted with the same volume of chloroform and centrifuged at $3000 \mathrm{rpm}$ for $15 \mathrm{~min}$. One milliliter of chloroform extract was removed and evaporated to dryness under vacuum. The residue was dissolved with $0.25 \mathrm{ml}$ of chloroform and the chloroform solution was subjected to analysis by gas chromatography.

Shimadzu model GC-4B gas chromatograph equipped with hydrogen flame ionization detector was used. A glass column ( $2 \mathrm{~m} \times 4 \mathrm{~mm}$ internal diam.) packed with $3 \% \mathrm{SE}-30$ on Chromosorb W DMCS was used at the following temperatures: column $240^{\circ} \mathrm{C}$, detector $300^{\circ} \mathrm{C}$, injector $300^{\circ} \mathrm{C}$. The carrier gas was $\mathrm{N}_{2}(40 \mathrm{ml} / \mathrm{min})$.

Ten milligrams per milliliter solution of Ic and IVb, and $1 \mathrm{mg} / \mathrm{ml}$ solution of IIIb in chloroform were used as internal calibration standards. The water solution of benzodiazepine mixtures, which contained known amounts of Ic, IVb and IIIb, was treated as described above and the accuracy of quantitative estimation was checked. The relative error was less than $10 \%$.

The gas chromatograms of authentic substances are shown in Fig. 1.

\section{RESULTS}

\section{Screening}

About 400 identified and unidentified

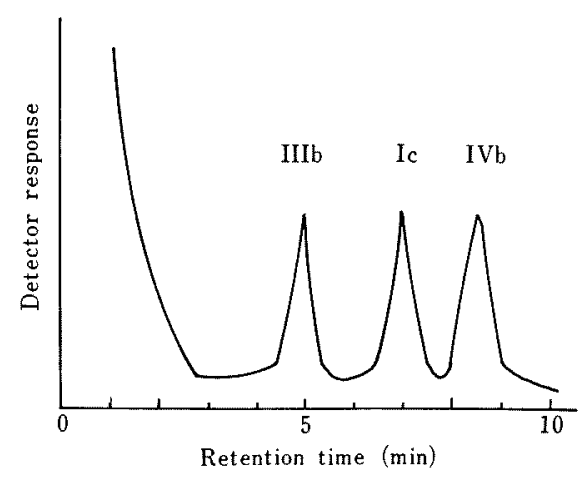

FIG. 1. Gas Chromatogram of Authentic Benzodiazepines.

Chromatographic conditions: column, $3 \%$ SE-30 on Chromosorb W DMCS in $4 \mathrm{~mm} \times 2 \mathrm{~m}$ glass tubing; nitrogen flows, $40 \mathrm{ml} / \mathrm{min}$; column temp., $240^{\circ} \mathrm{C}$; detector temp., $300^{\circ} \mathrm{C}$; injector temp., $300^{\circ} \mathrm{C}$. 


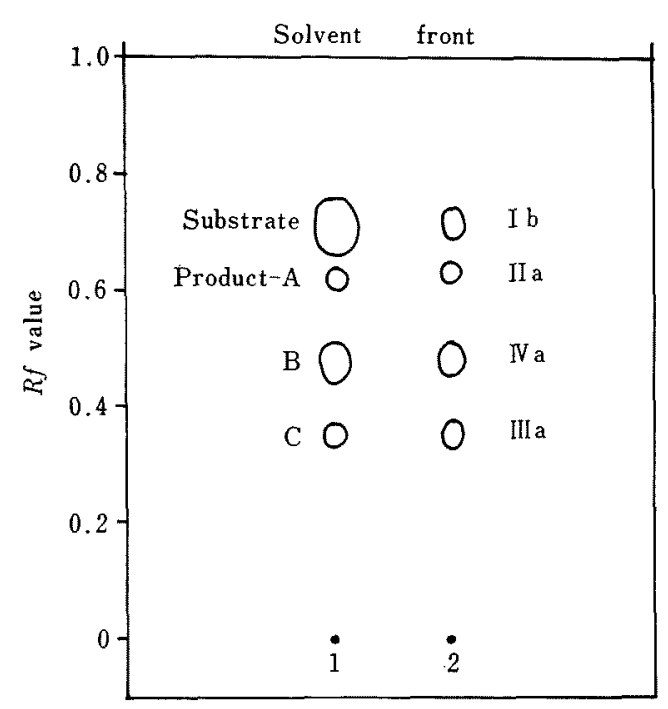

FIG. 2. Thin-layer Plate of Ib and Its Metabolic Products after 7 Days of Incubation of Ib with Streptomyces sp. S-60 or S-145.

1, Chloroform extract; 2 , synthesized authentic substances; solvent system, chloroform-acetone-ethanol $(17: 2: 1)$.

Streptomyces stock cultures were screened for their ability to hydroxylate benzodiazepines. Twenty cultures have been found to convert the compound $\mathrm{Ia}$ and $\mathrm{Ib}$ into the compoumd IVa and the compound Ic into IVb, but they did not formed the hydroxylated products.

However, ten cultures (Streptomyces sp. S-3, S-4, S-12, S-19, S-60, S-120, S-145, St. flavus ATCC 3369, St. griseochromogeneus ATCC 14,511 and St. chartreusis ATCC 14,922 ) have been found to convert the compound I into II and III, and compound IV into III. These ten cultures were used for transformation studies.

None of the cultures formed ferric chlorideferricyanide positive product.

\section{Transformation of prazepam (Ib) by Strepto- myces sp. $S-60$}

Two grams of Ib was incubated with S-60 for 7 days in twenty Sakaguchi flasks containing the SM-1 medium. As shown in Fig. 2, three products were detected on TLC plate. The substrate and three products were isolated by the procedures desctibed in MATERIALS AND

\section{Methods.}

One gram of substrate (Ib) was recovered.

Product-A (20 mg) was isolated as a wet crystalline substance which showed UV $\lambda_{\max }^{\mathrm{EtOH}}$ $\mathrm{nm}: 310,284$; IR $\nu_{m a x}^{\mathrm{Nujol}} \mathrm{cm}^{-1}: 3400(\mathrm{OH})$, 1700, 1680 (amide), 1600; NMR $\delta_{\text {TMS }}^{\mathrm{d}_{6} \text {-DMSO }}$ : $7.2 \sim 7.6(8 \mathrm{H}, \mathrm{m}, \mathrm{ArH}), 6.3(1 \mathrm{H}, \mathrm{d}, J=8 \mathrm{~Hz}$, disappeared with $\left.\mathrm{D}_{2} \mathrm{O}, \mathrm{CHO} \underline{\mathrm{H}}\right), 4.8(1 \mathrm{H}, \mathrm{d}$, $J=8 \mathrm{~Hz}$, became singlet with $\left.\mathrm{D}_{2} \mathrm{O}, \mathrm{CHOH}\right)$, $0.1 \sim 0.9$ (m, cyclopropyl). From these results, product-A was identified as 1-cyclopropylmethyl-7-chloro-1, 3-dihydro-3-hydroxy-5-phenyl-2H-1, 4-benzodiazepine-2-one (IIb).

Product-B (340 mg) was isolated as crystals, which after recrystalization from acetone, showed $\mathrm{mp} 215 \sim 217^{\circ} \mathrm{C}$; UV $\lambda_{\max }^{\mathrm{EtOH}} \mathrm{nm}: 318$; IR $\nu_{\text {max }}^{\mathrm{Nujol}} \mathrm{cm}^{-1}: 3150,3050(\mathrm{NH}), 1680$ (amide), 1600; NMR $\delta_{\mathrm{TMS}}^{\mathrm{CDCl}_{3}}: 9.5(1 \mathrm{H}, \mathrm{s}, \mathrm{NH}), 7.5(8 \mathrm{H}$, $\mathrm{m}, \mathrm{ArH}), 4.3\left(2 \mathrm{H}, \mathrm{s}, \mathrm{CH}_{2}\right)$. From these results, product-B was identified as 7-chloro-1, 3-dihydro-5-phenyl-2H-1, 4-benzodiazepine-2one (IVa).

Product-C (90 mg) was isolated as crystals, which after recrystalization from ethanol, showed $\mathrm{mp} 203 \sim 204^{\circ} \mathrm{C}$; UV $\lambda_{\max }^{\mathrm{EtOH}} \mathrm{nm}: 316$; IR $\nu_{\max }^{\mathrm{Nujol}} \mathrm{cm}^{-1}: 3340(\mathrm{OH}), 3200(\mathrm{NH}), 1700$, 1680 (amide), 1600; NMR $\delta \delta_{\text {TMS }}^{\mathrm{d}_{6} \text {-DMso }}: 10.5(1 \mathrm{H}$, s, NH), $7.6 \sim 7.3(8 \mathrm{H}, \mathrm{m}, \operatorname{ArH}), 6.4(1 \mathrm{H}, \mathrm{d}$, $J=8 \mathrm{~Hz}$, disappeared with $\mathrm{D}_{2} \mathrm{O}, \mathrm{CHOH}$ ), $4.9(1 \mathrm{H}, \mathrm{d}, I=8 \mathrm{~Hz}$, became singlet with $\mathrm{D}_{2} \mathrm{O}, \mathrm{CHOH}$ ). From these results, product-C was identified as 7-chloro-1, 3-dihydro-3hydroxy-5-phenyl-2H-1, 4-benzodiazepine-2one (IIIa).

Transformation of prazepam (Ib) by Streptomyces sp. $S-145$

Twelve grams of $\mathrm{Ib}$ was incubated with $\mathrm{S}-$ 145 for 6 days in two 10 liter jar fermentors containing the SM-1 medium. IIb (100 mg), IVa $(1.36 \mathrm{~g})$ and IIIa $(1.99 \mathrm{~g})$ were obtained and $1.6 \mathrm{~g}$ of $\mathrm{Ib}$ was recovered. The identity of these products were established by IR and NMR spectra.

Transformation of 7-chloro-1, 3-dihydro-5-(2'fluorophenyl)-2H-1, 4-benzodiazepine-2-one (IVb) by Streptomyces sp. S-145

Three grams of IVb was incubated with 
S-145 for 7 days in thirty Sakaguchi flasks containing the SM-1 medium. One product (product-D) was detected on TLC plate and it was isolated by the procedure described in Materials AND Methods.

Product-D $(80 \mathrm{mg})$ was isolated as crystals, which after recrystalization from ethanol, showed mp 193 $\sim 194^{\circ} \mathrm{C}$; UV $\lambda_{\max }^{\mathrm{EtOH}} \mathrm{nm}: 318$; IR $\nu_{m a x}^{\mathrm{Nufol}} \mathrm{cm}^{-1}: 3250(\mathrm{OH}), 3050(\mathrm{NH}), 1690$ (amide), 1610; NMR $\delta{ }_{\mathrm{TMS}}^{\mathrm{d}_{6} \text {-DMSO }}: 10.8(1 \mathrm{H}, \mathrm{s}$, $\mathrm{NH}), 7.7 \sim 7.1(7 \mathrm{H}, \mathrm{m}, \operatorname{ArH}), 6.2(1 \mathrm{H}, \mathrm{d}$, $J=8 \mathrm{~Hz}$, disappeared with $\left.\mathrm{D}_{2} \mathrm{O}, \mathrm{CHOH}\right), 4.8$ ( $1 \mathrm{H}, \mathrm{d}, J=8 \mathrm{~Hz}$, became singlet with $\mathrm{D}_{2} \mathrm{O}$, $\mathrm{CHOH}$. Anal. Found: C, 59.15; H, 3.36; N, 9.09; Cl, 11.41. Calcd. for $\mathrm{C}_{15} \mathrm{H}_{10} \mathrm{~N}_{2} \mathrm{O}_{2} \mathrm{ClF}$ : C, 59.13; H, 3.31; N, 9.19; Cl, 11.63. From these results, product-D was identified as 7-chloro-1, 3-dihydro-3-hydroxy-5-(2'-fluorophenyl)-2H-1, 4-benzodiazepine-2-one (IIIb). Recovery of substrate (IVb) was $2.5 \mathrm{~g}$.

Transformation of 7-chloro-1, 3-dihydro-1methyl-5-(2'-fluorophenyl)-2H-1, 4-benzodiazepine-2-one (Ic) by Streptomyces sp. $S-145$

Six grams of Ic was incubated with S-145 for 6 days in a 10 liter jar fermantor containing the SM-2 medium. Two products were detected on TLC plate and they were isolated by the procedures described in MATERIALS AND METHODS.

IVb (2.53 g) and IIIb (0.7 g) were obtained and $2.3 \mathrm{~g}$ of Ic was recovered. The identities of these products were established by IR and NMR spectra. In this experiment, IIc was not obtained.

Effects of culture conditions on the conversion of Ic or IVb into IIIb by Streptomyces sp. $S-145$

1. Medium. In order to find the suitable medium for the conversion of Ic or IVb into IIIb, many kinds of media were examined. Quantitative estimation of IIIb accumulated in fermentation broth was carried out by the procedures described in MAtERIALS AND Methods.

Bennet's medium and SM-3 medium were found to be favorable.
2. $p H$. The effect of initial $\mathrm{pH}$ on the conversion of Ic or IVb into IIIb was examined by the use of the SM-3 medium.

The initial $\mathrm{pH}$ of 7.0 appeared to be optimum.

3. Oxygen supply. The effect of oxygen supply on the conversion of IVb into IIIb was investigated in the shaking culture with the SM-3 medium at $50 \mathrm{ml}$ to $200 \mathrm{ml}$ in a $500 \mathrm{ml}$ Sakaguchi flask.

The highest conversion was obtained with $50 \mathrm{ml}$ of the medium. This result suggests that vigorous aeration is favorable for the conversion of $\mathrm{IVb}$ into IIIb.

4. Substrate concentration. The alcohol solution of IVb was added to the culture broth to give such concentrations as $0.03,0.05,0.1$, 0.2 and $0.5 \%(\mathrm{w} / \mathrm{v})$ in the total volume of 100 $\mathrm{ml}$.

The results indicated that the lower the substrate concentration, the larger the accumulation of IIIb in the culture broth and the higher the rate of conversion. The concentration of $0.05 \%$ was used for further experiments.

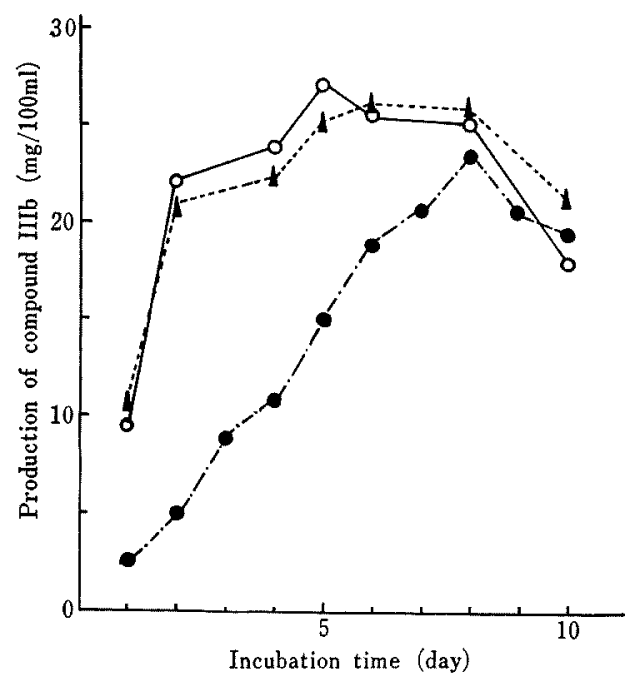

FIG. 3. Effect of Glycine on the Conversion of IVb into IIIb.

Basal medium: glucose $1 \%$, peptone $0.2 \%$, meat extract $0.1 \%$ and yeast extract $0.1 \%(\mathrm{pH} 7.0)$.

Cultivation: concentration of IVb was $0.05 \%$. Incubation was carried out at $28^{\circ} \mathrm{C}$ using $500 \mathrm{ml}$ Sakaguchi flask containing $100 \mathrm{ml}$ of the medium.

Concentration of glycine added to the medium: $\bullet-.-\bullet$, none; O- $0,0.05 \% ; \Delta-\cdots,-. \Delta, 0.1 \%$. 


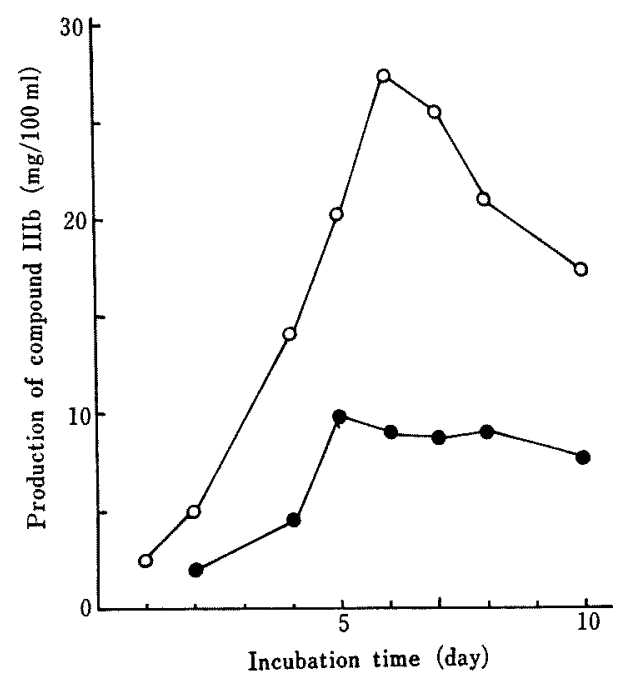

FIG. 4. Effect of Glycine on the Conversion of Ic into IIIb.

Culture conditions were the same as in Fig. 3 except for replacement of $\mathrm{IVb}$ by Ic.

Concentration of glycine added to the medium: - none; $0-0,0.05 \%$.

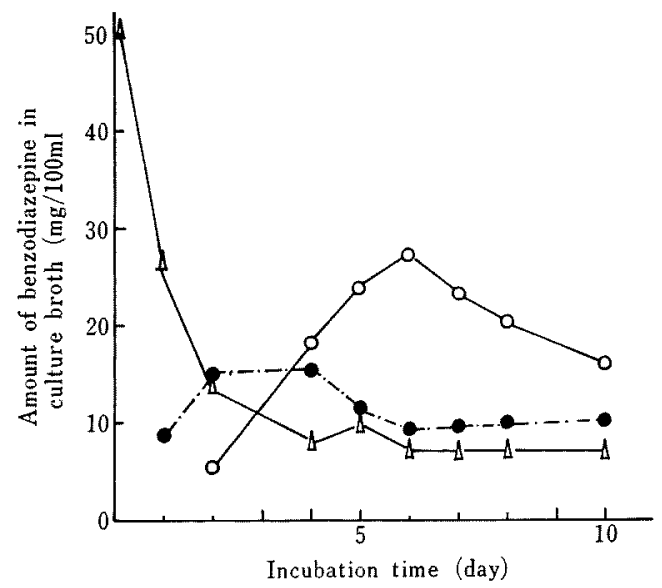

Fig. 5. Time Course of the Conversion of Ic into IVb and IIIb by Streptomyces sp. S-145.

Medium: glucose $1 \%$, peptone $0.2 \%$, meat extract $0.1 \%$, yeast extract $0.1 \%$ and glycine $0.05 \%$ pH 7.0 . Cultivation: seed culture $(120 \mathrm{ml})$, previously grown for 2 days, was inoculated into 10 liter jar fermentor containing 6 liters of the medium. After incubation at $28^{\circ} \mathrm{C}$ for 1 day, $3 \mathrm{~g}$ of Ic was added and further incubation was carried out. Seven liters of air per min was introduced into fermentor.

$\triangle \longrightarrow \triangle \mathrm{Ic} ; 0-\cdot \mathrm{OV}, \mathrm{IV} ; \mathrm{O}-\mathrm{O}, \mathrm{IIIb}$.

5. Effect of glycine. The effect of glycine on the conversion of IVb into IIIb was ex- amined. As shown in Fig. 3, the addition of glycine to the medium increased the accumulation of IIIb in the culture broth but a difference between 0.05 and $0.1 \%$ of glycine concentration was not apparent. Figure 4 shows the effect of glycine on the conversion of Ic into IIIb. The addition of glycine at a concentration of $0.05 \%$ increased considerably the accumulation of IIIb in the culture broth.

\section{Time course of conversion of Ic into IVb and $I I I b$}

Figure 5 shows the time course of conversion of Ic into IVb and IIIb by Streptomyces sp. S-145. Substrate (Ic) decreased rapidly and thereafter the amount of Ic remaining in the broth was approximately constant. The compound IVb was formed and reached maximum level in a relatively short time and thereafter the amount of IVb accumulating in the broth was approximately constant. The amount of IIIb accumulating in the broth reached maximum on the 6th day and decreased thereafter.

Transformation of Ic with 100 liter jar fermentor

Seed culture of Streptomyces sp. S-145 (1.4 liters), previously grown for 2 days, was inoculated into 100 liter jar fermentor containing 70 liters of the SM-2 medium. After incubation at $28^{\circ} \mathrm{C}$ for 1 day, $35 \mathrm{~g}$ of Ic and $100 \mathrm{ml}$ of soy bean oil were added and further incubation was carried out for 6 days. Seventy liters of air per min was introduced into the fermentor. The products were isolated by the procedures described in Materials and Methods.

Four grams of Ic ( $11 \%$ recovery), $10 \mathrm{~g}$ of IVb $(30 \%$ conversion) and $9.5 \mathrm{~g}$ of IIIb $(28 \%$ conversion) were obtained.

\section{DISCUSSION}

Greenspan et al. ${ }^{1 \prime}$ reported that Pellicularia flamentosa f. sp. microsclerotia CBS transformed diazepam into 3-hydroxy derivatives and a quinazolinone derivative and that $P$ filamentosa f. sp. microsclerotia IFO 6298 transformed diazepam into 3-hydroxy derivatives and 4-oxide derivatives. 
In the present studies, ten strains of Streptomyces species transformed diazepam into 3hydroxy derivatives. In addition, it was found that various $2 \mathrm{H}-1$, 4-benzodiazepine-2-ones were hydroxylated in the 3-position of the seven-membered ring rather than in the aromatic rings and that not only S-60 and S145 but eight other strains also formed 3hydroxylated products from various $2 \mathrm{H}-1$, 4-benzodiazepine-2-ones. 4-Oxide derivatives and quinazolinone derivatives were not obtained.

When 1-alkyl-2H-1, 4-benzodiazepine-2ones, such as Ia and Ib, were used for the substrates, dealkylated products were formed in the beginning. Then, hydroxylation of the dealkylated products was carried out and at last some hydroxylation of the substrates seemed to occur. 1-Alkyl-3-hydroxy-2H-1, 4-benzodiazepine-2-ones were not obtained under certain conditions. Based on these results, possible metabolic pathways of $2 \mathrm{H}-1$, 4-benzodiazepine-2-ones in Streptomyces are shown in Scheme 1.

Liver microsomal oxidations of a variety of substrates are known to be mediated by a mixed function oxygenase system. In the case of tertiary amines, it has been proposed that microsomal oxygenation leads to the formation of a carbinolamine via tertiary amine $\mathrm{N}$.
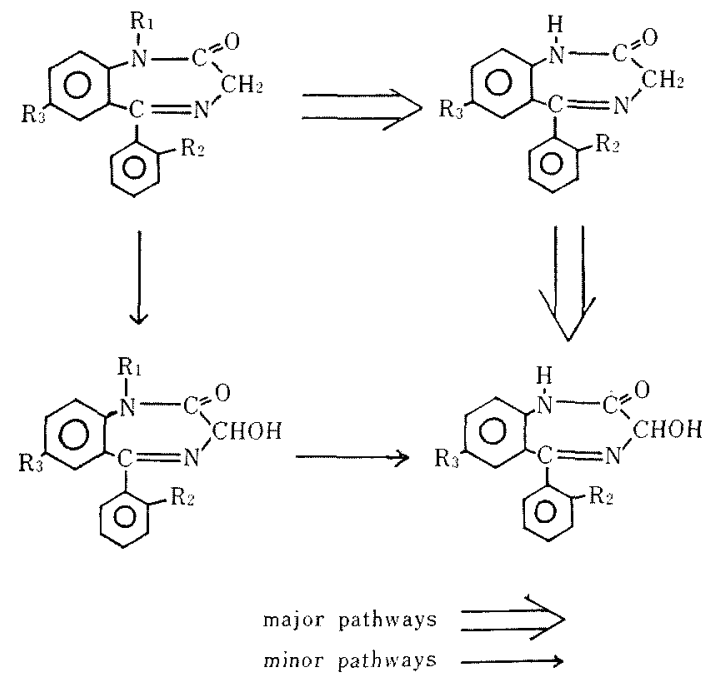

SCHEME 1. oxide. $^{5 \sim 8)}$ Bell et $a l^{3,9)}$ have shown that 7chloro-1, 3-dihydro-5-phenyl-2H-1, 4-benzodiazepine-2-one 4-oxide was converted into 3-acetoxy-7-chloro-1, 3-dihydro-5-phenyl-2H-1, 4-benzodiazepine-2-one with acetic anhydride. These facts indicate the possibility that Noxide type intermediate participates in enzymatic hydroxylation of $2 \mathrm{H}-1$, 4-benzodiazepine-2-ones. However, Sadee et al. ${ }^{10)}$ reported that diazepam 4-oxide was not detected in the incubate of diazepam with rat liver microsomes and that it was not transformed to 3-hydroxy derivative with rat liver microsomes.

In our studies, 4-oxide derivatives were not obtained and 7-chloro-1, 3-dihydro-5-phenyl2H-1, 4-benzodiazepine-2-one 4-oxide added to the broth was not converted into the 3 hydroxy derivative. These facts suggest that the formation of 3-hydroxy-2H-1, 4-benzodiazepine-2-ones is not carried out via 4-oxide derivatives.

As shown in Fig. 4, glycine was extensively effective for the conversion of Ic into IIIb by Streptomyces sp. S-145. One of the main actions of glycine may be protection against bactericidal action of the compound Ic, for Streptomyces sp. S-145 had an inclination to lyse with this compound in the absence of glycine.

Acknowledgement. The authors wish to thank Dr. H. Wada and Mr. S. Inaba of our laboratories for their helpful advice and encouragement.

\section{REFERENCES}

1) G. Greenspan, H. W. Ruelius and H. E. Alburn, U. S. Patent, 3453179 (1969).

2) Japan Patent, 45-28187.

3) S. C. Bell and S. J. Childress, J. Org. Chem., 27, 1961 (1962).

4) A. Stempel, I. Douvan, E. Reeder and L.H. Sternboch, ibid., 32, 2417 (1967).

5) J. R. Gillette, Advan. Pharmacol., 4, 219 (1966).

6) M. H. Bickel, Pharmacol. Rev., 21, 325 (1969).

7) E. Wada and K. Yamasaki, J. Am. Chem. Soc., 76, 155 (1954).

8) D. M. Ziegler and F. H. Pettit, Biochem. Biophys. Res. Commun., 15, 188 (1964).

9) S. C. Bell, T. S. Sulkowski, C. Gochman and S. I. Childress, J. Org. Chem., 27, 562 (1962).

10) W. Sadee, W. Garland and N. Castangnoli, $J$. Med. Chem., 14, 643 (1971). 